\title{
A model of so-called "Zebra" emissions in solar flare radio burst continua
}

\author{
R. A. Treumann ${ }^{1,2, *}$, R. Nakamura ${ }^{3}$, and W. Baumjohann ${ }^{3}$ \\ ${ }^{1}$ Department of Geophysics and Environmental Sciences, Munich University, Munich, Germany \\ ${ }^{2}$ Department of Physics and Astronomy, Dartmouth College, Hanover NH 03755, USA \\ ${ }^{3}$ Space Research Institute, Austrian Academy of Sciences, Graz, Austria \\ *visiting: International Space Science Institute, Bern, Switzerland
}

Received: 12 April 2011 - Revised: 20 August 2011 - Accepted: 21 September 2011 - Published: 29 September 2011

\begin{abstract}
A simple mechanism for the generation of electromagnetic "Zebra" pattern emissions is proposed. "Zebra" bursts are regularly spaced narrow-band radio emissions on the otherwise broadband radio continuum emitted by the active solar corona. The mechanism is based on the generation of an ion-ring distribution in a magnetic mirror geometry in the presence of a properly directed field-aligned electric potential field. Such ion-rings or ion-conics are well known from magnetospheric observations. Under coronal conditions they may become weakly relativistic. In this case the ion-cyclotron maser generates a number of electromagnetic ion-cyclotron harmonics which modulate the electron maser emission. The mechanism is capable of switching the emission on and off or amplifying it quasi-periodically which is a main feature of the observations.
\end{abstract}

Keywords. Solar physics, astrophysics, and astronomy (Radio emissions) - Space plasma physics (Radiation processes; Wave-particle interactions)

\section{Introduction}

Substantial effort has been invested into the explanation of an apparently minuscule effect, the so-called "Zebra bursts", in the radio emission from some objects. They are best known from the Sun to occur during broadband continuum radio bursts like, for instance, type IV and type I burst radio continua where they have been detected first. The frequencytime spectrogram of a typical (non-drifting) example in the metric wavelengths is shown in Fig. 1. It is by now quite well established that these radio bursts are frequently (if not regularly) caused in the aftermath of solar flares by the electroncyclotron maser emission from the anisotropic magnetically

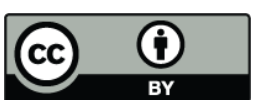

Correspondence to: R. A. Treumann (rudolf.treumann@geophysik.unimuenchen.de) trapped flare-electron population. "Zebras" break the radio continuum by organising it into regularly drifting or undulating narrow-band patterns in the frequency-time spectrograms with strictly equally spaced emission and absorption features. A typical "Zebra" emission looks like a fingerprint on the otherwise unstructured or irregularly structured radio comtinuum. This fingerprint can be stationary or inclined, i.e. as a whole exhibiting a positive or negative frequency drift. An excellent and exhaustive review of the various occasions when and types of such drifting or stationary "Zebra" patterns can, for instance, be found in Chernov (2010), a paper which reliefs us from going more into detail on the observations. Though for the energy budget of the radiation "Zebras" are indeed minuscule and thus of little importance, their observation is nevertheless important as they provide some deep insight into the complicated plasma processes during flares and the generation of radio continua.

The quasi-harmonic frequency spacing of the spectral "Zebra" fine structures which appear as narrow-band multiharmonic modulations (emission/absorption structures up to thirty harmonics have been reported) has sometimes been suspected to be related to the ion cyclotron frequency. However, no convincing mechanism of how they could be produced has so far been found. In the past a number of mechanisms have also been proposed which search for explaining these fine structures differently (as for an example see, e.g. LaBelle et al., 2003) as representations of inherent properties of the electron radiation or other nonlinear processes like wave-wave interactions. Neither of these mechanisms has, however, convincingly demonstrated the harmonic spacing or the sequence of emissions and absorptions which is frequently observed. The pros and contras for the various models have been listed and discussed in the above cited paper by Chernov (2010).

We should note at this occasion that similar ion-cyclotron modes can also be generated without the direct involvement of relativistic ion beams. This could happen when ion

Published by Copernicus Publications on behalf of the European Geosciences Union. 


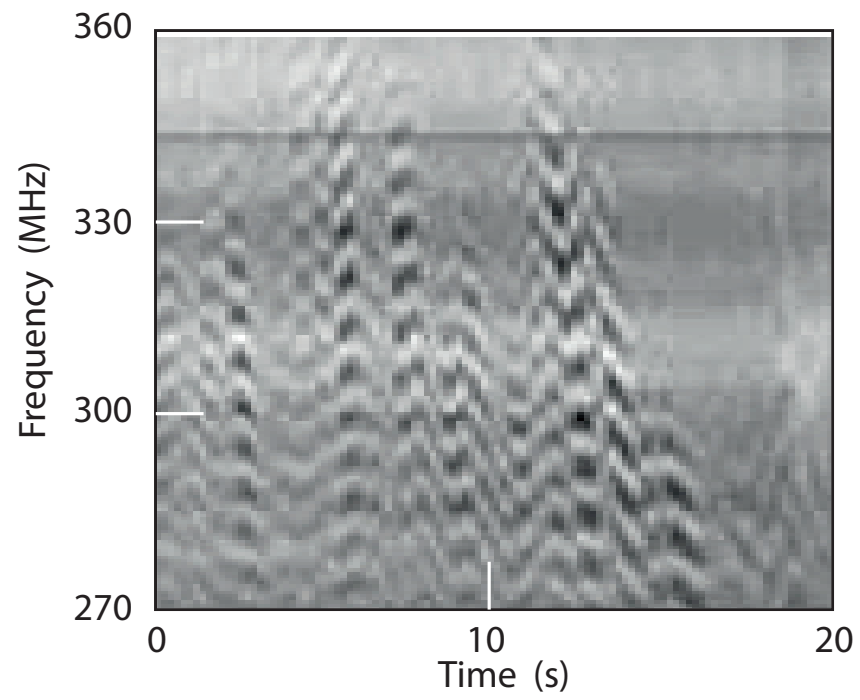

Fig. 1. A typical example of a non-drifting Zebra emission pattern on a weakly variable solar radio continuum background. Shown is the frequency-time spectrogram of the a brief part of the originally observed emission (data taken from Chernov, 2010). We have chosen only $20 \mathrm{~s}$ of observation and cut out $90 \mathrm{MHz}$ in frequency in the metric wavelengths range. The typical Zebra pattern comes out clearly on the linear frequency scale consisting of many equally spaced chains of consecutive emission and absorption bands. The radio antenna seems to snapshot different emission sites at different times and for different length of time. Some such sites exhibit frequency drifts in either direction from high to low but also from low to high frequencies. When emissions from two (or more) sites are detected at same time as on the right, then patchy structures are created according to the constructive or destructive interference of emissions.

holes evolve on the ion plasma background in the presence of strong field-aligned currents as a non-linear state of ionacoustic waves. Such ion holes will be subject to the action of the ion-cyclotron maser and generate harmonics of the ion cyclotron frequency in a way similar to that recently explored (Treumann et al., 2011) for the electron-cyclotron maser emission from electron holes in view of the explanation of radiation fine structure observed in the auroral kilometric radiation spectra.

Here we exploit the very simple idea that the "Zebra" emissions fine structure in the solar flare radio continuum could indeed be a consequence of the presence of electromagnetic ion-cyclotron harmonics. These ion-cyclotron harmonics are genuinely caused by an high energy ion distribution which propagates on the radiating electron background. The ion beam in such a case will itself radiate ion cyclotron waves which propagate on the R-X mode branch. Because of their frequencies $\omega \sim \ell \omega_{\mathrm{ci}} \ll \omega_{\mathrm{ce}}$ (with harmonic number $\ell=1,2,3 \ldots$..) being much less than the electron cyclotron frequency $\omega_{\text {ce }}$, they can however not leave the plasma. This is

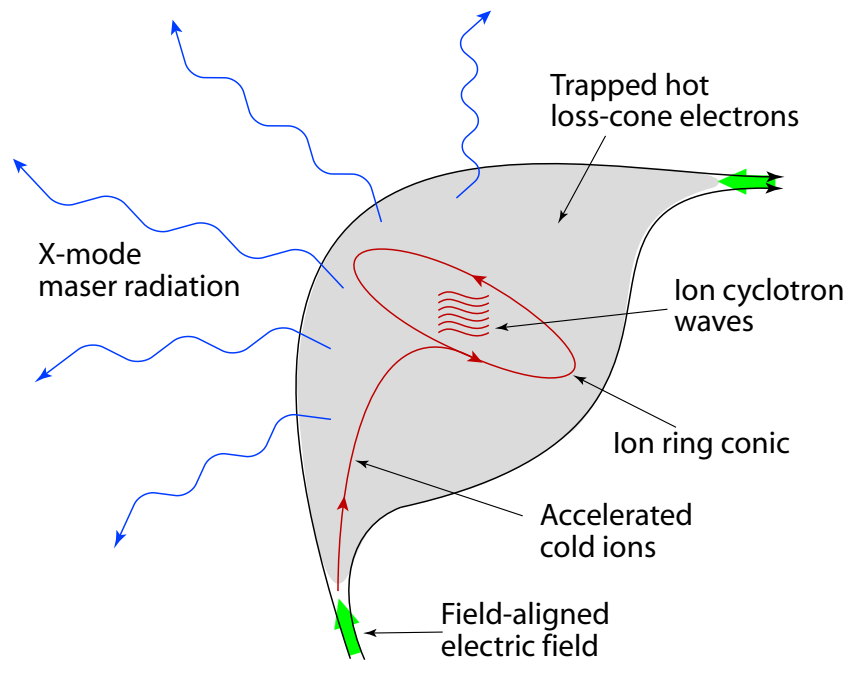

Fig. 2. The model of a post-flare magnetically trapped electron distribution which is the source of type IV electron cyclotron maser radiation. In this stage the weakly relativistic hot electrons have evolved into a loss-cone distribution of Dory-Guest-Harris type with completely empty sharply edged loss cone assuming that whistler activity has ceased (simply because $\omega_{\text {pe }} / \omega_{\text {ce }} \ll 1$ ) and quasilinear diffusion into the loss cone is terminated. The R-X mode emission is caused on the section of the resonance circle in phase space that cuts through the steep perpendicular phase space gradient of the loss cone and is mostly perpendicular to the magnetic field at frequency below but very close to the local electron cyclotron frequency. Upward directed strong electric fields at the site of the magnetic mirror accelerate cold ions into an ion-conic distribution which propagates up into the configuration and generates large numbers of ion cyclotron harmonics. The local modulation of the electron loss cone distribution by the ion wave spectrum modulates the electron cyclotron maser and causes the genuine ionharmonic spaced "Zebra" emissions.

in contrast to the electron radiation which is emitted into free space. Hence, a mechanism must be found which imprints the ion structure on the radiation generated by the electrons in order to make it visible. Such a mechanism is given by the interaction with the electron background. Under certain restrictive conditions this interaction causes a modulation of the electron maser which leads to the observed "Zebra" fine structure.

Justification of such a model (as is shown in Fig. 2) can be given by reference to the well-established flare reconnection model. According to this model flares are caused by magnetic reconnection in the lower corona at field strengths of several Gauss $\lesssim B \lesssim$ few 100 Gauss. In this process localised magnetic field-aligned electric potential drops develop which accelerate and heat the flare electrons and accelerate ions until they evolve into high energy essentially cold conical phase space distributions, a process well-known from auroral physics. Under solar flare radio continuum conditions 
the ions readily become relativistic. Their cold conical distributions excite ion-cyclotron harmonic waves via the ioncyclotron maser instability.

It may be in place to elaborate a little more on the model even though it digresses from the main purpose of the present paper which focusses on the multi-ion-cyclotron harmonic modulation of the electron maser radiation. In a mirror magnetic field geometry like that of Fig. 2 (or some its variants) which has been generated in the course of the flare by reconnection, say, on its lower left part. The electrons in this configuration have immediately developed a loss-cone distribution having lost that part of the distribution inside the loss cone. These electrons escaped along the magnetic field and went down into the solar chromosphere where they cause $\mathrm{X}$-rays. Some electrons have also been accelerated into a fast dilute electron beam along the reconnection separatrix. These excite electron holes and other high-frequency plasma waves seen sporadically as type I and type III bursts. They do not interest us here.

Reconnection implies but fairly strong localised fieldaligned electric fields. These fields when acting on the ions accelerate the ions along the magnetic field depending on the direction of the electric field. At the location where the field is directed into the trapped configuration a fast and cold ion beam results which moves up into the magnetic trap and mirrors at the opposite side (as shown in the figure). In combination with the magnetic mirror force $-\mu_{\mathrm{i}} \nabla_{\|} B$ and further conservation of the magnetic moment $\mu_{\mathrm{i}}$ of the ions, however, the mirroring ion beam evolves into an ion-conic (Chiu and Schulz, 1978) transferring the acceleration in the mirroring into perpendicular velocity. At the weak magnetic fields in the magnetosphere reconnection accelerates ions just up to energies of some $10 \mathrm{MeV}$. In the strong coronal magnetic fields the resulting ion conic beam will have relativistic energy in the $\mathrm{GeV}$ range which in the lower corona cause gamma-rays.

Ion conics are thus mirroring ion velocity distributions with ion loss cone and lacking particles with small parallel velocities. They are thus moving ion rings. The extreme case is a standing ion ring with zero parallel speed $u_{\|}=0$. If it is cold, then all ions have the same perpendicular "beam" velocity $u_{\perp}=U_{\mathrm{ib}}$. In the Earth's magnetosphere ion conics have been observed almost continuously (as for a few references the reader may consult André and Yau, 1997; Miyake et al., 1996; Shelley, 1995).

\section{Ion-cyclotron maser instability}

The cyclotron maser is a relativistic instability that involves the presence of at least weakly relativistic particles. It had been originally proposed for electrons only, first in the construction of free-electron lasers (Motz, 1951; Madey, 1971) and masers before being recognised for plasmas (by Twiss, 1958 , in its non-relativistic non-efficient version) and, much later (in its relativistic version by Wu and Lee, 1979), as a very efficient radiation mechanism in space plasmas as well (for a review cf. Treumann, 2006). Subsequently it has been identified as the main radio wave emission process in the aurora of Earth and other planets, in solar radio bursts, particularly type I, type IV and so-called microwave (or $\mu$-) bursts, and under astrophysical conditions.

The electron cyclotron maser as the main emission mechanism in all these objects is well established by now. Below we will make use of it. However, as for the "Zebra" fine structure which interests us here, the electron cyclotron maser is just the carrier of information. Adopting the notion that the "Zebra" pattern maps the ion cyclotron frequency into the emitted electron maser radiation, we propose that an exactly similar mechanism works as well for the ions causing an ion-cyclotron maser instability. Such an instability has, in fact, been proposed first by Hoshino and Arons (1991) and was refined by Amato and Arons (2006) to include a cold relativistic gyrating ion beam, i.e. the extreme case of a standing cold ion conic. In a different non-relativistic version it was used in space plasma (cf. e.g. Chaston et al., 2002) where it should serve to excite harmonics of low frequency electromagnetic ion cyclotron waves in the auroral plasma.

For efficient generation of ion cyclotron harmonic waves under radio continuum radiation conditions (type I, type IV, microwave-bursts etc.) one requires the presence of a relativistic gyrating ion distribution with anisotropic velocity and positive perpendicular phase space gradient. For simplicity, the relativistic model ion-conic distribution is taken to be cold and anisotropic

$F_{\mathrm{ib}}\left(u_{\perp}, u_{\|}\right)=\frac{1}{2 \pi U_{\mathrm{ib}}} \delta\left(u_{\perp}-U_{\mathrm{ib}}\right) \delta\left(u_{\|}\right)$

where now $u$ is the normalised 4-velocity $[u=\gamma \beta$ with $\gamma=\left(1-\beta^{2}\right)^{-\frac{1}{2}}=\left(1+u^{2}\right)^{\frac{1}{2}}$ the Lorentz factor], and $U_{\mathrm{ib}}$ is the initial value of $u$ for the relativistic ions, and subscripts $\perp, \|$ refer to the direction of the magnetic field. For electromagnetic waves with perpendicular wave vector $\boldsymbol{k}=k \hat{x}$ the dispersion relation becomes

$n^{2}=\epsilon_{y y}-\epsilon_{x y} \epsilon_{y x} / \epsilon_{x x}, \quad \epsilon_{x y}=-\epsilon_{y x}, \quad n^{2} \equiv k^{2} c^{2} / \omega^{2}$

The explicit form of the dielectric tensor components is standard and has been given e.g. by Amato and Arons (2006) whose results we will make use of here and who use a pair plasma as background.

In order to exploit their results we have to re-scale their parameters to conditions of an electron-ion background. These parameters are the ratios $\sigma=B^{2} / \mu_{0} m N c^{2} \gamma$ of magnetic to kinetic energy densities for the different species (in their case, the ion beam and background electron and positron pairs, index \pm , for which they use numerically $\sigma_{\mathrm{ib}}=$ $10^{-2}, \sigma_{ \pm}=2$ ). We replace the positrons with ions and use the quasi-neutrality condition $N=N_{\mathrm{e}}=N_{\mathrm{i}}+N_{\mathrm{ib}}$, where the subscript "ib" denotes the relativistic ring ions. This yields for 


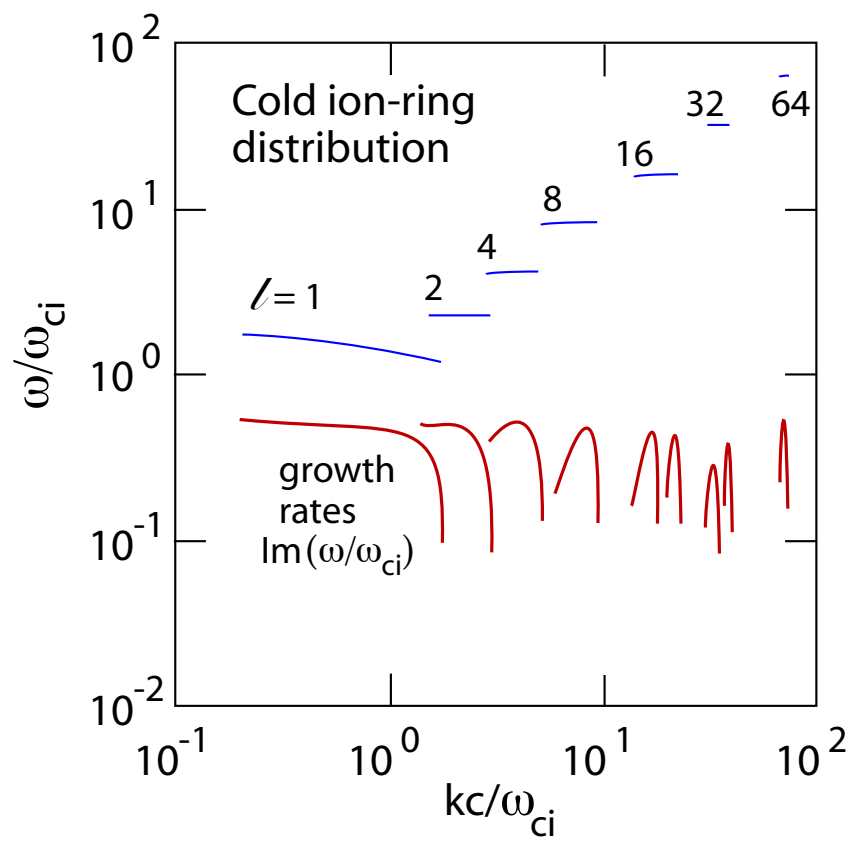

Fig. 3. Ion-cyclotron maser dispersion relation. Harmonic frequencies (blue) and growth rates (red) excited by a cold relativistic proton-ring distribution in an electron-proton plasma with ringLorentz factor $\gamma_{\mathrm{ib}}<10, N_{\mathrm{ib}} / N \sim 10^{-2}$ (re-scaled data taken from Amato and Arons, 2006). The ring-ion maser generates a large number of ion cyclotron harmonics $\ell$, all of nearly same maximum growth rate. The ion-cyclotron harmonics are equally spaced. In this figure, however, we have chosen for a logarithmic frequency scale in order to show a large number of harmonics and their growth rates. In order not to overload the figure we plotted only selected harmonics $\ell=2^{n}, n \in \mathrm{N}$.

the background ion Lorentz factor $\gamma_{\mathrm{i}}=\mu \gamma_{\mathrm{e}} /\left(1-N_{\mathrm{ib}} / N\right) \approx$ $\mu \gamma_{\mathrm{e}}$.

For small beam densities $N_{\mathrm{ib}} \ll N$, with $\mu=m_{\mathrm{e}} / m_{\mathrm{i}}$, the background ions are non-relativistic, and only the radiating electrons will be considered relativistic. Moreover, we have $\gamma_{\mathrm{ib}} / \gamma_{\mathrm{e}} \sim 200 \mu\left(N / N_{\mathrm{ib}}\right)$. Since the electron background is assumed mildly relativistic we will have $\gamma_{\mathrm{ib}} \sim 0.1\left(N / N_{\mathrm{ib}}\right) \gamma_{\mathrm{e}} \lesssim$ 10 for applying the results of Amato and Arons (2006). With these quite reasonable numbers we can directly refer to Fig. 13a of Amato and Arons (2006) the re-scaled version of which we reproduce here as Fig. 3.

One observes that a very large number of electromagnetic ion cyclotron harmonics is generated which propagate on the $\mathrm{R}$-X mode branch. (One may note that only harmonics $\ell=2^{n}$ are shown for clarity; clearly the harmonics on a linear frequency scale are spaced at equal distance.) These waves have all roughly the same growth rate and are of narrow bandwidth. These harmonics are practically all confined to the plasma even in an underdense plasma under the condition

$\omega_{\mathrm{e}}^{2} / \omega_{\mathrm{ce}}^{2}<2 T_{\mathrm{e}} / m_{\mathrm{e}} c^{2}<1$

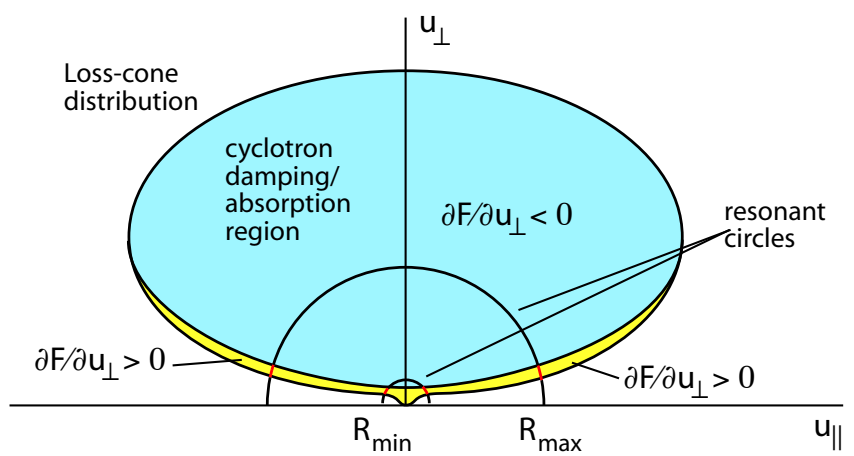

Fig. 4. Electron loss-cone (Dory-Guest-Harris) distribution in the normalised 4-velocity plane. Shown are two resonance circles for maximum and minimum radii, corresponding to minimum and maximum unstable electron cyclotron maser emissions in perpendicular propagation of $\mathrm{R}-\mathrm{X}$ - modes. The section of the resonance circles contributing to emission are shown in red. Emission is provided as long as the phase space gradient over the positive (yellow) part is steep enough to compensate for the larger part of the circles traversing the blue negative gradient region of the distribution. Note that the blue region between the circles of maximum and minimum resonances $R_{\max }, R_{\min }$ absorbs electron-cyclotron waves by damping them. This heats the electrons in this domain and flattens the distribution in this region of phase space.

of the electron-cyclotron maser instability (Winglee, 1983) as long as their frequency is below the upper hybrid frequency $\omega_{\mathrm{uh}} \gtrsim \omega_{\mathrm{ce}}$ in this case. The key observation is that for the trapped radiating electrons the perpendicular electric fields

$E_{\perp}(t)=\sum_{\ell} E_{\ell} \exp \left(-i \ell \omega_{\mathrm{ci}} t\right)$

of these ion cyclotron harmonics are practically stationary electric fields which generate drift motions in the magnetically trapped anisotropic electron background plasma that is responsible for the radio continuum. These electron drifts modulate the emitted electron cyclotron maser radiation, adding factors $2 \pi \delta\left(\omega \pm \ell \omega_{\mathrm{ci}}\right)$ to the emission spectrum.

\section{Electron-cyclotron maser radiation}

We briefly indicate what kind of variation in the spectrum of the electron cyclotron emission is to be expected in this case. The continuum radiation model as for instance a type IV is based on the assumption of a magnetically trapped electron distribution. Such distributions are of loss-cone type which can, for instance, be modelled as (relativistic) Dory-GuestHarris distributions

$F_{\mathrm{e}}\left(u_{\perp}, u_{\|}\right)=\frac{1}{\pi^{\frac{3}{2}} v_{\mathrm{e}}^{3} s !}\left(\frac{u_{\perp}^{2}}{v_{\mathrm{e}}^{2}}\right)^{s} \exp \left[-\frac{u_{\perp}^{2}+u_{\|}^{2}}{v_{\mathrm{e}}^{2}}\right], \quad s \in \mathrm{R}$ 
where $v_{\mathrm{e}}^{2}$ is the normalised thermal spread, and the factor $u_{\perp}^{2 s} \propto \sin ^{2 s} \theta$, with $\theta$ the angle between 4-velocity and magnetic field direction, accounts for the loss cone. Note that $s$ can be any real number while being most conveniently chosen as $s \in \mathrm{N}$.

With the above distribution function the instability has been exhaustively discussed by Pritchett (1986) for different propagation angles finding that largest growth is obtained for strictly perpendicular propagation, $k_{\|}=0$. In this case the resonance condition for resonant excitation of waves becomes

$u_{\perp}^{2}+u_{\|}^{2}-2\left(1-v_{\mathrm{ce}}\right)=0$

where $v_{\mathrm{ce}}=\omega / \omega_{\mathrm{ce}}$ is the non-relativistic ratio of wave frequency to electron cyclotron frequency. Clearly, resonance is possible only for $v_{\text {ce }} \lesssim 1$ and, as usual, the resonance line is a circle in the normalised 4-velocity plane $\left(u_{\perp}, u_{\|}\right)$of radius

$R_{\mathrm{res}}=\sqrt{2\left(1-v_{\mathrm{ce}}\right)}$

located between the minimum and maximum radii $R_{\min }, R_{\max }$ of resonance which correspond to maximum and minimum resonant frequencies, respectively (see Fig. 4).

The relativistic cold plasma dispersion relation of the R-X mode, which is the real part of

$n^{2}-1-\left(2-n_{\perp}^{2}\right) A=0, \quad$ with $\quad n^{2}=k^{2} c^{2} / \omega^{2}$

allows for a range of such resonant frequencies below the non-relativistic $\omega_{\mathrm{ce}}$ which is quite narrow (cf. e.g. Pritchett, 1986), depending on the plasma parameters. The maximum resonance frequency (minimum radius) is very close to $v_{\mathrm{ce}}=1$. On the other hand, the minimum resonance frequency can be estimated from the cold X-mode dispersion relation yielding

$v_{\mathrm{ce}, \min } \approx 1-\frac{\gamma_{\mathrm{e}}^{2}}{2}\left(\frac{\omega_{\mathrm{e}}}{\omega_{\mathrm{ce}}}\right)^{2}$

For consistency we note that this condition is in accord with Eq. (3) which requires that $\omega_{\mathrm{ce}}^{2}>\omega_{\mathrm{pe}}^{2}$, i.e. an underdense plasma. Type IV radiation is emitted at, say, frequency $\omega / 2 \pi \sim 10^{8}-10^{10} \mathrm{~Hz}$, preferentially closer to the higher of these frequencies. Acknowledging that this is at the local electron-cyclotron frequency, then it corresponds to magnetic fields of the order of $B \sim$ several $100 \mathrm{G}$, and the electron-cyclotron maser operates for densities $N \sim$ $10^{10} \mathrm{~cm}^{-3}$ or less, typical for the lower corona and higher.

The function $A$ contains the plasma response. Taking $k_{\|}=0,\left|1-v_{\text {ce }}\right| \ll 1$, one has to first order $\operatorname{Re}(A) \ll 1$. This expression is to be used in the calculation of the growth rate

$\operatorname{Im}\left(v_{\mathrm{ce}}\right) \simeq-\frac{1}{2} \operatorname{Im}(A)$
Maser radiation will be produced if $\operatorname{Im}(A)<0$ is negative (corresponding to "negative absorption" of the electromagnetic R-X mode). The resonant imaginary part of $A$ is given by

$$
\begin{aligned}
\operatorname{Im}(A)= & -\frac{\pi^{2}}{2 v_{\mathrm{ce}}} \frac{\omega_{\mathrm{e}}^{2}}{\omega_{\mathrm{ce}}^{2}} \int_{-\infty}^{\infty} \int_{0}^{\infty} \mathrm{d} u_{\|} u_{\perp} \mathrm{d} u_{\perp}^{2} \frac{\partial F_{\mathrm{e}}}{\partial u_{\perp}} \times \\
& \times \delta\left(u_{\|}^{2}+u_{\perp}^{2}-R_{\mathrm{res}}^{2}\right)
\end{aligned}
$$

In dealing with the loss cone distribution Fig. 4 one realises that any substantial modulation of the particle distribution caused by the presence of the ion cyclotron waves is important mainly when it affects the low perpendicular energy electrons at the loss cone boundary (red piece of resonance circle in the figure). These electrons are particularly vulnerable to the $\boldsymbol{E} \times \boldsymbol{B}$ drift that is caused by the low frequency ion-cyclotron waves. Since this is the only region which has positive perpendicular gradient and therefore contributes to wave growth over the narrow part of the boundary crossed by the resonant circle, its modulation modulates the electron cyclotron maser. The longer part of the resonance circle (blue in the figure) inside the distribution sees a weak negative gradient and causes wave absorption which for instability is grossly over-compensated by the very steep gradient at the loss-cone boundary.

Inspection of Fig. 5 suggests, that the modulation of the loss-cone boundary indeed heavily affects the emission conditions. In presence of ion-cyclotron waves the lowperpendicular energy electrons at the loss-cone boundary will start oscillating up and down in $u_{\perp}$ with the imposed frequency of the ion cyclotron wave electric field, when performing an $\boldsymbol{E}_{\perp} \times \boldsymbol{B}$-drift. This drift displaces the loss-cone boundary either up to higher $u_{\perp}$ or down. In the latter case the loss-cone is partially filled, and the maser may switch off, causing zero emission intensity at the respective frequency. In the former case the loss-cone widens, faking a larger loss-cone exponent $s$ and bringing the minimumradius resonance almost completely into the positive gradient region which should amplify the emission at frequency very close to $v_{\text {ce }} \sim 1$. These two cases are shown schematically in Fig. 5.

Including this modulation effect into the calculation of the electron cyclotron maser instability from scratch on is quite involved. We can however boldly construct a simple model which possesses some of the relevant features of the modulation.

Realising that the two time scales of the ion cyclotron and electron instabilities are quite far apart, we consider the modulation as stationary on the time scale of the electron cyclotron maser emission. Then, the drift effect will result in a modulation of the loss cone as has been described above and can be included by replacing $u_{\perp} \rightarrow u_{\perp}^{\prime}=u_{\perp}+\Delta$ in the 

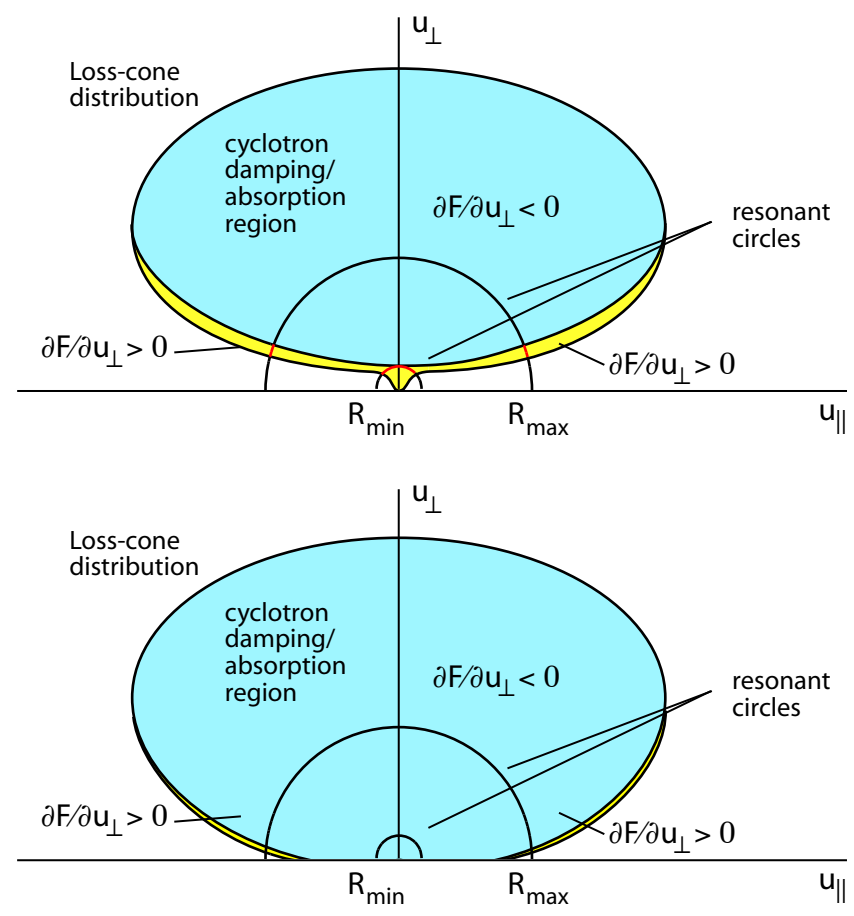

Fig. 5. Same as in Fig. 4 except for the modulation of the loss cone included. Top: the modulation due to the electric field drift in the transverse electric field of the ion-cyclotron mode shifts the perpendicular velocity up thereby opening the loss cone and pushing its gradient to higher $u_{\perp}$. This steepens the gradient and reduces the damping part of the resonance circle. At minimum radius (largest emission frequency $v_{\mathrm{ec}}$ ) the resonance circle is fully in the positive gradient. During this phase emission is amplified. Bottom: during this phase the drift reduces the perpendicular velocity filling the loss cone and switching the maser off.

loss-cone factor of the electron distribution function Eq. (5), where

$\Delta=\gamma_{\mathrm{e}} V_{E}(t) / c=\gamma_{\mathrm{e}} \beta_{E}$

is the contribution of the normalised drift in the ion-cyclotron wave electric field. Here $\beta_{E}(t)$ is the slowly variable relativistic velocity caused by the ion-cyclotron fields. Though this is small, its effect can nevertheless be large as the maser is extremely sensitive to modulation of the loss-cone.

Modulation can, in addition, also be included as a variation of the loss-cone exponent $s$ replacing it with a variable exponent $s \rightarrow a(s, t)>0$ and $t$ the slow time scale, allowing for a direct response of the loss cone to the modulation. Since $a$ is not anymore an integer number, this latter step requires that in the normalisation of the DGH distribution Eq. (5) the factor $s$ ! is replaced by the Gamma-function $\Gamma(a+1)$. Performing the derivative yields

$$
\frac{\partial F_{\mathrm{e}}}{\partial u_{\perp}}=\frac{2}{u_{\perp}^{\prime}}\left(a-\frac{u_{\perp} u_{\perp}^{\prime}}{v_{\mathrm{e}}^{2}}\right) F_{\mathrm{e}}
$$

Inserting into Eq. (11), performing the integration with respect to $u_{\perp}^{2}$, rearranging and neglecting terms higher than first order in $\Delta$ reduces to

$$
\begin{aligned}
& \operatorname{Im}(A) \approx-\frac{\sqrt{\pi} R_{\mathrm{res}}}{\Gamma(a+1)} \frac{\omega_{\mathrm{e}}^{2}}{v_{\mathrm{ce}} \omega_{\mathrm{ce}}^{2}} \frac{\mathrm{e}^{-R_{\mathrm{res}}^{2}}}{v_{\mathrm{e}}^{2}} \int_{0}^{1} \frac{x^{a} \mathrm{~d} x}{\sqrt{1-x}}\left[\left(\frac{a}{R_{\mathrm{res}}^{3}}-x\right.\right. \\
& \left.\left.+\frac{\Delta^{\prime}}{R_{\mathrm{res}}} x^{\frac{1}{2}}\right)+\Delta^{\prime}(2 a+1)\left(\frac{a}{R_{\mathrm{res}}} x^{-\frac{1}{2}}-x^{+\frac{1}{2}}\right)\right]
\end{aligned}
$$

where $\Delta^{\prime}=\sqrt{1+R_{\text {res }}^{2}} V_{E}(t) / c$. All quantities are normalised as indicated earlier. (In order to arrive at the above expression we have taken $R_{\text {res }}$ out and changed the fake integration variable.) The integral can be evaluated making use of $\int_{0}^{1} \mathrm{~d} x^{a} / \sqrt{1-x}=\mathrm{B}\left(a+1, \frac{1}{2}\right)$, where $\mathrm{B}(x, y)=$ $\Gamma(x) \Gamma(y) / \Gamma(x+y)$ is the beta-function and $\Gamma\left(\frac{1}{2}\right)=\sqrt{\pi}$. This yields

$\operatorname{Im}(A) \approx-C\left(Q_{1}+\Delta^{\prime} Q_{2}\right)$

where $C \equiv\left(\pi R_{\text {res }} \omega_{\mathrm{e}}^{2}\right) \mathrm{e}^{-R_{\text {res }}^{2}} /\left(v_{\text {ce }} \omega_{\text {ce }}^{2} v_{\mathrm{e}}^{2}\right)>0$. The term $Q_{1}$ depends solely on the loss-cone exponent $a$, while $Q_{2}$ depends on $a$ and accounts for the variation in the perpendicular velocity. These functions are

$$
\begin{aligned}
Q_{1} & =\frac{1}{\left(a+\frac{1}{2}\right) \Gamma\left(a+\frac{1}{2}\right)}\left[\frac{a}{R_{\mathrm{res}}^{3}}-\frac{a+1}{a+\frac{3}{2}}\right], \\
Q_{2} & =\frac{a(2 a+1) \Gamma\left(a+\frac{1}{2}\right)}{(a+1) \Gamma^{2}(a+1)}\left[\frac{1}{R_{\mathrm{res}}}-a\right]
\end{aligned}
$$

\subsection{Modulation by the induced cross-field drift $\Delta$}

Observing that always $a \geq 0$ we first neglect $Q_{1}$. Then, $Q_{2}$ [and $\operatorname{Im}(A)]$ changes sign at $a=R_{\text {res }}^{-1}=\left[2\left(1-v_{\mathrm{ce}}\right)\right]^{-\frac{1}{2}}$. If $a=s$ is an integer, this means that at sufficiently large loss cones and for $\Delta^{\prime}>0$ the term with $Q_{2}$ damps the resonance and could theoretically cause absorption. On the other hand, since $\left|v_{\mathrm{ce}}-1\right| \ll 1$ this requires quite large $a$. We reasonably assume that $a<R_{\text {res }}^{-1}$ and thus $Q_{2}>0$. However, the sign of this term also depends on $\Delta^{\prime} \propto V_{E} \propto \sin \left(\ell \omega_{\mathrm{ci}} t\right)$ which oscillates with harmonic ion cyclotron frequency $\ell \omega_{\mathrm{ci}}$. As we have proposed, this causes a sinusoidal modulation of the term including $Q_{2}$ and leads to modulation of the electron maser emission at the resonance frequency. At small $a$, however, we have always $Q_{2}<Q_{1}$. In order for the second term in $\operatorname{Im}(A)$ to dominate one requires that with $\beta_{E}^{m}=\left|V_{E}^{\max } / c\right|$

$$
\left(2 a R_{\mathrm{res}}^{2} \gamma_{\mathrm{e}} \beta_{E}^{m}\right)^{-1}<\frac{\Delta^{\prime}}{\gamma_{\mathrm{e}} \beta_{E}^{m}} \approx\left|\sin \left(\ell \omega_{\mathrm{ci}} t\right)\right|<1
$$

where, for simplicity, we assumed just one sinusoidal ioncyclotron harmonic belonging to the entire spectrum of ion waves that are generated by the cold ion conic distribution. The argument of $\sin ^{-1}$ must be smaller than one. Hence this condition, combined with the condition on $a$

$R_{\mathrm{res}}^{-1}>a \gg\left(2 \gamma_{\mathrm{e}} \beta_{E}^{m}\right)^{-1}$ 
and with $\gamma_{e} \gtrsim 1$ implies that

$R_{\text {res }}<2 \beta_{E}^{m} \quad$ or $\quad 1-v_{\text {ce }} \ll 2\left(\beta_{E}^{m}\right)^{2}$

These conditions can be satisfied only for sufficiently steep loss cone gradients $a$ and large drifts caused by the ioncyclotron harmonics. If this is the case, the growth rate Eq. (15) of the electron-cyclotron maser instability is periodically modulated during the phases of the ion-cyclotron harmonics. It is amplified during positive phases $0<\omega_{\mathrm{ci}} t<\pi$ and switched off during negative phases $\pi<\omega_{\mathrm{ci}} t<2 \pi$. The frequency of this modulation of the electron-cyclotron maser radiation at the local resonance is proportional to the ion cyclotron frequency $\omega_{\mathrm{ci}}$.

Though the modulation is small in the growth rate, it modulates the exponential amplification factor of the maser emission which causes a much stronger exponential modulation of the emitted radiation intensity. However, it requires a sufficiently relativistic trapped electron component with steep loss cone gradient in addition to the presence of a cold relativistic ion ring passing across the electron background.

\subsection{Emitted power spectrum}

In order to directly see the modulation, we calculate the power emitted by the electron-cyclotron maser at resonant frequency $v_{\mathrm{ce}}$. Poynting's theorem yields

$\mathcal{P}\left(v_{\mathrm{ce}}, \tau_{\mathrm{esc}}\right)=\frac{1}{\mu_{0} c}\left|E_{\perp}\left(v_{\mathrm{ce}}\right)\right|_{0}^{2} \mathrm{e}^{\left[2 C\left(Q_{1}+\Delta^{\prime} Q_{2}\right) \tau_{\mathrm{esc}}\right]}$

where we have assumed that $\omega_{\mathrm{ce}} v_{\mathrm{ce}} / k \simeq c$.

We are interested only in the modulation of the power. We therefore do not calculate the power at saturation which requires the precise knowledge of the saturation process. Since in the collisionless case the radiation can presumably freely escape this requires knowledge of the competition between cyclotron maser emission and cyclotron re-absorption along the ray path. This is not impossible to estimate but lies outside the scope of our investigation. So we stay with the linear state, assuming that the power is limited just by escape from the volume, i.e. by the time $\tau_{\mathrm{esc}} \sim L / c$, where $L$ is of the order of the linear dimension of the trapped electron volume perpendicular to the magnetic field (see Fig. 2).

The first term in the exponential accounts for the amplitude the power can reach, the second term accounts for the cyclotron modulation via the time dependence of $\Delta^{\prime} \propto$ $\sin \left(\ell \omega_{\mathrm{ci}} t\right)$, where the time $t$ is now measured on the scale of the ion-cyclotron period. Evaluating the exponential term, we find for

$\mathrm{e}^{2 C \tau_{\mathrm{esc}} \Delta^{\prime} Q_{2}}=I_{0}(z)+2 \sum_{\eta=1}^{\infty} I_{\eta}(z) \exp \left[i \eta\left(\frac{\pi}{2}-\ell \omega_{\mathrm{ci}} t\right)\right]$

where $I_{\eta}(z)$ is the modified Bessel function of order $\eta$, and $z=2 \tau_{\mathrm{esc}} C Q_{2} \beta_{E}^{m} \sqrt{1+R_{\mathrm{res}}^{2}}$. One observes that the term $I_{0}(z)$ on the left does not produce any modulation. Since the power is a real quantity, only the real part of this expression contributes. Thus we obtain

$\mathcal{P}\left(v_{\mathrm{ce}}, \tau_{\mathrm{esc}}, t\right)=\mathcal{P}_{0}\left\{I_{0}(z)+\sum_{\eta=1}^{\infty} I_{\eta}(z) \sin \left[\eta\left(\ell \omega_{\mathrm{ci}} t\right)\right]\right\}$

where $(\ell, \eta) \in \mathrm{N}$, and we have written

$\mathcal{P}_{0}=\frac{1}{\mu_{0} c}\left|E_{\perp}\left(v_{\mathrm{ce}}\right)\right|_{0}^{2} \exp \left[2 C Q_{1} \tau_{\mathrm{esc}}\right]$

These expressions show indeed that the emitted maser power experiences a modulation at multiples of the ion cyclotron frequency. In frequency space $\varpi$, defining $\xi=\ell \omega_{\mathrm{ci}}$, this corresponds to

$$
\begin{aligned}
\mathcal{P}\left(v_{\mathrm{ce}}, \varpi\right) & \propto 2 \pi \mathcal{P}_{0}\left\{I_{0}(z) \delta(\varpi)+\right. \\
& \left.+\sum_{\eta=1}^{\infty}\left|I_{\eta}(z)[\delta(\varpi-\eta \xi)-\delta(\varpi+\eta \xi)]\right|\right\}
\end{aligned}
$$

The first term on the right describes just the stationary spectrum while the sum superimposes the ion cyclotron modulation on the spectrum.

\subsection{Modulating the loss-cone boundary gradient $a$}

So far we considered the modulation induced by the addition of a perpendicular drift velocity $\Delta$ to the original loss cone velocity. This model essentially produced a quasi-periodic shift of the loss cone boundary as a whole causing a quasiperiodic sequence of filling and emptying the loss cone. One immediately realises that such a model must compete with the loss process. This model thus applies only when the filling of the loss-cone proceeds on a faster time scale than the very loss process. In other words it assumes weak losses of electrons on the time scale of the ion-cyclotron frequency, i.e. $\tau_{\text {loss }} \omega_{\mathrm{ci}}>1$. Only in this case the loss cone remains filled for half a cyclotron period. Assuming that binary collisions are responsible for electron loss, the condition for application of the former model becomes $\omega_{\mathrm{ci}}>v_{\mathrm{en}}$ where $v_{\mathrm{en}} \approx n_{\mathrm{n}} \sigma_{\mathrm{c}} v_{\mathrm{e}}$ is the electron collision frequency with the neutral component, $n_{\mathrm{n}}$ is the neutral density, $\sigma_{\mathrm{c}} \approx 5 \times 10^{-15} \mathrm{~cm}^{2}$ the collisional cross section, and $v_{\mathrm{e}}$ the electron speed.

In the opposite case when the losses are strong, i.e. $\omega_{\mathrm{ci}} \tau_{\text {loss }}<1$, the loss cone is emptied almost instantaneously, i.e. faster than the ion cyclotron frequency can modulate the loss cone width. Under such conditions similar effects can be caused by modulating the steepness of the loss cone boundary. It is in fact the steepness of the loss-cone boundary gradient which most strongly affects the emission of radiation through modulating the perpendicular phase space gradient of the electron distribution function. One realises this from inspection of the resonance condition $R_{\text {res }}$ and from a short glance at Fig. 4. It is only the narrow sections of the resonance circle which contribute to emission. Hence modifying the perpendicular velocity gradient at the loss-cone boundary 


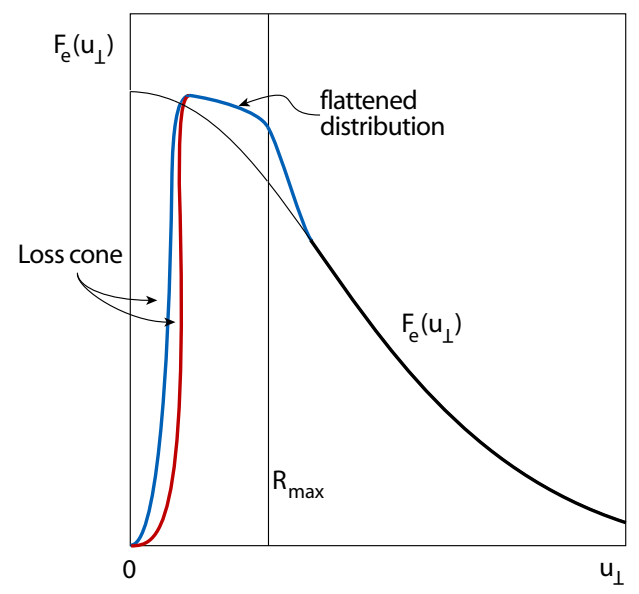

Fig. 6. Illustration of the widening of the loss cone by shifting the lower energy particles. The original Maxwellian is shown as a thin line. Cyclotron absorption (cf. Fig. 4) of the part of the distribution inside the maximum resonant radius $R_{\max }$ flattens the distribution. Shift of the loss cone may either flatten or steepen the loss cone gradient $a$.

$\left(\partial F_{\mathrm{e}} / \partial u_{\perp}\right)_{\mathrm{lcb}}$ should have a strong effect on the electroncyclotron maser emission rate and power. This gradient is controlled by the loss-cone exponent $a$ as can be seen from Fig. 6.

The loss-cone exponent $a \geq 0$ cannot be negative. Among the infinite possibilities of modelling $a$ we choose

$a=a^{\prime} \sin ^{2}\left(\ell \omega_{\mathrm{ci}} t\right) \geq 0$

Thus the power oscillates within the bounds $0 \leq a \leq a^{\prime}$, where $a^{\prime}=s \Delta^{\prime 2}$. One first observes that setting $a=0$, which corresponds to no loss cone, immediately yields $Q_{1}<$ $0, Q_{2}=0$, and the electron-cyclotron maser switches off, as it should in the absence of a loss cone. Thus, we need to consider only the behaviour of $Q_{1}$. This is done quickly, with the result that

$a=0$ for $\omega_{\mathrm{ci}} t=\pi, \quad$ and $\quad \ell \in \mathrm{N}$

in which case the maser switches periodically off and on at half the ion-cyclotron harmonic-oscillation period.

We note that for real positive argument, $0<[\Gamma(x)]^{-1}<\frac{3}{2}$; the inverse Gamma function has no poles and no zeros for $x \neq 0$, maximises at $x \approx \frac{3}{2}$, and for $x>2$ decreases monotonically. Since in our case $x=a+\frac{1}{2}$, the inverse Gamma function behaves regularly and does not introduce any problems. Hence, any periodic modulation of the loss-cone power like the one given in our choice of $a$ will cause a modulation of the electron cyclotron maser emission at the resonant frequency over the entire (narrow) bandwidth $v_{\mathrm{ce}, \min } \leq v_{\mathrm{ce}} \leq$ $v_{\text {ce, max }}$ of the electron-cyclotron maser emission.

It can also be shown in passing that for the only allowed small values of $R_{\text {res }}^{3}$, the function $Q_{1}$ (and thus also the growth rate of the electron cyclotron maser) are monotonic functions lacking any maximum. This can be seen when taking the differential of $Q_{1}$ with respect to $a$. Defining $\bar{a} \equiv a_{\max }$ we obtain the transcendent equation for $\bar{a}$

$$
\left(\bar{a}+\frac{1}{2}\right) \Gamma\left(\bar{a}+\frac{1}{2}\right) \approx\left(\bar{a}-\frac{1}{2}\right)^{\frac{4}{5}}(\bar{a}+2)^{\frac{1}{5}}
$$

which for $a>0$ has no solution. Also, if not neglecting $R_{\text {res }}$, the maximum condition can be written as

$\Gamma\left(\bar{a}+\frac{3}{2}\right) \approx\left(\bar{a}+\frac{3}{2}\right)^{\frac{2}{5} R_{\mathrm{res}}^{3}} \mathrm{e}^{\bar{a}}$

which has no solution either for $a>0$.

Another kind of modulation occurs, in addition, under the condition that $Q_{1}$ changes sign at $a \lesssim \frac{2}{3} R_{\text {res }}^{3}$. This implies that the maser is switched off when the phase of the sinus assumes the value $\ell \omega_{\mathrm{ci}} t<\sin ^{-1} \sqrt{2 R_{\text {res }}^{3} / 3 a^{\prime}}$. This may be realised at increasingly shorter ion-cyclotron harmonic phases

$\omega_{\mathrm{ci}} t \sim \ell^{-1} \sin ^{-1} \sqrt{2 R_{\mathrm{res}}^{3} / 3 a^{\prime}}$

Since the argument of $\sin ^{-1}$ must always be smaller than 1 , this kind of modulation can occur only if $a^{\prime}=s \Delta^{\prime 2}>2 R_{\text {res }}^{3} / 3$ which corresponds to an initial steepness of the loss cone gradient

$s>\frac{2}{3} R_{\text {res }}^{3} \beta_{E}^{-1}$

where as before $\beta_{E}=V_{E} / c$. Since both, $\beta_{E}$ and $R_{\text {res }}$ are small numbers, the above product partially compensates. Still, $s$ has to be sufficiently large initially in order to allow for this kind of modulation, and if the right-hand side of the above condition is small. In the opposite case $Q_{1}>0$ the electron-cyclotron maser will be active, and the dominant modulation effect will be of the kind given in Eq. (27) due to the periodic vanishing of $a \rightarrow 0$.

Within the simple model used for the time dependence of $a(t)$, the maser emission caused by a given ion-cyclotron harmonic $\ell$ is modulated with frequency $\varpi=2 \ell \omega_{\mathrm{ci}}=2 \xi$. Of course, other models of the loss cone angle and loss cone exponent may lead to different modulation frequencies, but within the most simple assumptions of our model we find a periodic modulation of the electron cyclotron maser emission of the kind needed in so-called "Zebra" bursts.

\section{Conclusions}

The above mechanism, a sketch of which is given for illustration in Fig. 2, may occasionally work and produce the wanted "Zebra" structures with periodic amplification and suppression of electron cyclotron maser emission in continuum radio bursts. It requires a rather steep or abrupt transition between the electron distribution and the loss cone. Two such mechanisms have been proposed, one holding for weak losses 
from the loss cone, the other for almost immediate losses. They also depend on the excitation of strong ion cyclotron harmonic waves in order to satisfy the restrictions imposed. This assumes that an intense ion (conic) ring distribution has been generated during the flare, a process that works only in the presence of sufficiently strong field aligned electric fields produced in a magnetic mirror configuration. Because of these reasons it might not be realised very frequently. It might, however, be realised occasionally. Yet, the presence of very high energy ions in solar flares is well known from the observation of gamma rays that are caused in nuclear processes. These ions are beamed during the flare process by the reconnection electric field along the magnetic field and are probably further accelerated in wave-prticle interactions. For the mechanism proposed here it suffices that part of the accelerated relativistic protons passes along the magnetic field across the trapped electron distribution. We have described that they will evolve into a conic by the combined action of the acceleration and mirror processes in which case they excite the ion-cyclotron maser. Modulation of the electron losscone boundary then becomes inevitable with the effects on the electron cyclotron maser emission described. The radiation becomes quasi-periodically amplified and attenuated in this case simultaneously in many ion-cyclotron harmonics. Overlap may cause very complicated interference patterns in a dynamic frequency-time radio spectrogram. On the other hand, the occasional observation of very clear harmonic " $\mathrm{Ze}$ bras" suggests that one observes a single radiation site, a flux tube section crossed by the ion beam. This is of substantial interest because "Zebras" themselves being energetically minuscule serve as probes of the state of the flare plasma.

Clearly the proposed model of "Zebra" emissions in solar burst radio continua is not unique. It depends on the choice of a model for the electric field of the ion-cyclotron waves which causes the electric cross-field drift of the electrons which are responsible for the emission of the background solar burst radiation continuum. It depends moreover on the choice of the loss-cone distribution; choosing another analytical form of the loss-cone than the Dory-Guest-Harris distribution will introduce other parameters in place of $a$ and $\Delta$ the form, variation and effects of which must then be investigated along similar lines as in this paper.

One may easily imagine other models as well, for instance the involvement of electrostatic ion cyclotron harmonic (EICH) waves (instead of the electromagnetic ion cyclotron harmonics) which are known to be excited in the auroral kilometric radiation source above the Earth up to comparably high harmonics in the presence of the auroral counter streaming electron and ion beams. They are as well local being restricted to the location of the magnetic field-aligned potential drop which accelerates both electrons and ions into opposite directions along the magnetic field causing the ion conics which have been used in our model.

Here the Dory-Guest-Harris loss-cone distribution has been chosen because, in contrast to other proposed loss-cone distributions, it is always positive and conserves the particle number without additional constraints. Its selection was also guided for the convenience it provides in taking the perpendicular phase space derivative $\partial F / \partial u_{\perp}$ in the relativistic case. We are well-aware that it is just a model distribution which is not derived from first principles but includes some hidden process that is responsible for the generation of a losscone, i.e. the loss process of electrons in a magnetic mirror configuration. Loss-cone distributions are by no means necessarily Dory-Guest-Harris distributions.

What concerns the uncritical application of the DoryGuest-Harris distribution to the relativistic case, so we are very well aware that a more precise choice would have been starting from some version of the Maxwell-Jüttner distribution, which is the exact Boltzmann distribution in special relativity. This would require constructing the equivalent losscone distribution from it, which is not easily possible. In our case - as in all publications on the electron-cyclotron maser mechanism - we have just used its weakly relativistic version where the relativistic factor $\gamma$ can be expanded up to the required order with respect to the 4-velocity $u$. In this case the distribution we used is exact also in the relativistic case, within the above mentioned limits of the validity of the Dory-Guest-Harris distribution.

However, in spite of all the noted caveats, the general observation will still remain unchanged and valid: that the modulation of the loss-cone, in particular the quasi-periodic modulation of the perpendicular gradient of the loss-cone edge, will necessarily cause a modulation of the electroncyclotron maser. In our case it may modulate and also switch the electron-cyclotron maser on and off imposing temporary periodicity on the emitted power of the order of the $\ell$-th ioncyclotron harmonics $\ell \omega_{\text {ci }}$ and multiples of it. Locally this produces the typical "Zebra" emission/absorption bands.

One may wonder why in a large volume, for instance like that of a type IV, these harmonics are not washed out spatially and thus also in frequency space. This is, however, not a question specific to "Zebra" structures. Rather it is a general problem of any of the various fine structures in radio continua; in its generality there exists no unambiguous answer.

From our point of view, "Zebra" fine structures are probably locally generated and do not distribute over the large volume of the radio continuum. For instance, if they occur in the extended weak magnetic field region of the magnetic trap, the maser frequency may be nearly constant along the ion path. Chernov (2010) has listed a number of fine structures which he all attributes to the same family of "Zebra" emissions, some of them drifting upward or downward in frequency space, others each emission consisting of temporarily periodically arranged emission spots and so on.

Whether all these emissions belong to one and the same family and whether they can all be explained by the "Zebra" mechanism, is not known. Common features between some of them are their periodicities. So fibers might be taken 
as inclined (or drifting) "Zebras" (as suggested by Chernov, 2010) in spatially varying magnetic fields, still being local emissions which are related to the presence of fast cold ion conics, while the spotted "Zebra" emissions might be caused by switching on/off effects of the above kind. Here we do not enter into any discussion of these fine structures. The purpose of the present communication was just to present a simple model of the connection between electromagnetic ion-cyclotron waves and the electron-cyclotron maser emission mechanism capable of generating the observed ordinary "Zebra" emissions in radio continua.

Acknowledgements. This research was part of an occasional Visiting Scientist Programme in 2006/2007 at ISSI, Bern. RT thankfully recognises the assistance of the ISSI librarians, Andrea Fischer and Irmela Schweizer. He highly appreciates the encouragement of André Balogh, former Director at ISSI.

Topical Editor R. Forsyth thanks two anonymous referees for their help in evaluating this paper.

\section{References}

Amato, E. and Arons, J.: Heating and nonthermal particle acceleration in relativistic, transverse magnetosonic shock waves in proton-electron-positron plasmas, Astrophys. J., 653, 325-338, doi:10.1023/A:1004921619885, 2006.

André, M. and Yau, A.: Theories and observations of ion energization and outflow in the high latitude magnetosphere, Space Sci. Rev. 80, 27-48, doi:10.1086/508050, 1997.

Chaston, C. C., Bonnell, J. W., McFadden, J. P., Ergun, R. E., and Carlson, C. W.: Electromagnetic ion cyclotron waves at proton cyclotron harmonics, J. Geophys. Res., 107, 1351, doi:10.1029/2001JA900141, 2002.

Chernov, G. P.: Recent results of zebra patterns in solar radio bursts, Res. Astron. Astrophys., 10, 821-866, doi:10.1088/16744527/10/9/002, 2010.
Chiu, Y. T. and Schulz, M.: Self-consistent particle and parallel electrostatic field distributions in the magnetosphericionospheric auroral region, J. Geophys. Res., 83, 629-642, doi:10.1029/JA083iA02p00629, 1978.

Hoshino, M. and Arons, J.: Preferential positron heating and acceleration by synchrotron maser instabilities in relativistic positron-electron-proton plasmas, Phys. Fluids B, 3, 818-833, doi:10.1063/1.859877, 1991.

LaBelle, J., Treumann, R. A., Yoon, P. H., and Karlicky, M.: A model of Zebra emission in solar type IV radio bursts, Astrophys. J., 593, 1195-1207, doi:10.1086/376732, 2003.

Madey, J. M. J.: Stimulated emission of bremsstrahlung in a periodic magnetic field, J. Appl. Phys., 42, 1906-1913, doi:10.1063/1.1660466, 1971.

Miyake, W., Mukai T., and Kaya, N.: On the origins of the upward shift of elevated (bimodal) ion conics in velocity space, J. Geophys. Res., 101, 26961-26969, doi:10.1029/96JA02601, 1996.

Motz, H.: Applications of the radiation from fast electron beams, J. Appl. Phys., 22, 527-535, doi:10.1063/1.1700002, 1951.

Pritchett, P. L.: Electron cyclotron maser instability in relativistic plasmas, Phys. Fluids, 29, 229-315, doi:10.1063/1.865492, 1986.

Shelley, E. G.: The auroral acceleration region: The world of beams, conics, cavitons, and other plasma exotica, Rev. Geophys. Suppl., 33, 709-714, 1995.

Treumann, R. A.: The electron-cyclotron maser for astrophysical application, Astron. Astrophys. Rev., 13, 229-315, doi:10.1007/s00159-006-0001-y, 2006.

Treumann, R. A., Baumjohann, W., and Pottelette, R.: Electroncyclotron maser radiation from electron holes, Ann. Geophys., in preparation, available at: arXiv:1106.0274v1 [physics.spaceph], 2011.

Twiss, R. Q.: Radiation transfer and the possibility of negative absorption in radio astronomy, Austr. J. Phys., 11, 564, doi:10.1063/1.865492, 1958.

Winglee, R. M.: Interrelation between azimuthal bunching and semirelativistic maser cyclotron instabilities, Plasma Phys., 25, 217-255, doi:10.1088/0032-1028/25/2/006, 1983.

Wu, C. S. and Lee, L. C.: A theory of the terrestrial kilometric radiation, Astrophys. J., 230, 621-626, doi:10.1086/157120, 1979. 